\title{
An intracranial tumor appeared two months after the operation of bladder cancer: metastatic or primary?
}

\begin{abstract}
It is common for an intracranial mass to occur after another malignancy, which is usually metastasis. There are also reports that intracranial tumors have appeared years after another carcinoma had been previously treated with radiotherapy or chemotherapy. In the latter case, the intracranial tumor is usually attributed to metastasis or a secondary primary malignancy. There are also some hereditary or familial syndromes that present with multiple tumors, e.g., Li Fraumeni, Lynch, and Turcot syndrome. For these syndromes, the proband cancer generally occurs in children and young adults with a definitive familial aggregation. However, we found no previous reports of the appearance of a primary glioma only 2 months after a bladder cancer carcinectomy in an old patient without a typical family history. Here, we report the case of a 59-year-old female who complained of a constant headache for one week at the time of admission. Only 2 months before, she had undergone a bladder cancer carcinectomy. Upon admission, a cranial MRI scan revealed right temporal lobe lesions, and the post-operative pathological diagnosis was anaplastic astrocytoma. Cases such as these are easily misdiagnosed, and the opportunity for surgical treatment can be lost. Moreover, the appearance of two concurrent cancers or the occurrence of one after another within a very short interval in different body parts has rarely been reported. Therefore, this case may provide neurosurgeons with knowledge of an uncommon clinical experience that could reduce misdiagnosis and mistreatment.
\end{abstract}

Keywords: glioma, bladder cancer, cancer concurrence, secondary primary malignancy
Volume 7 Issue 2 - 2017

\author{
Congyan Wu, Yaodong Zhao \\ Department of Neurosurgery, Shanghai General Hospital, China
}

Correspondence: Yaodong Zhao, Department of

Neurosurgery, Shanghai General Hospital, China, I Haining Road, Shanghai, China, Post code: 28, Tel 862 I36I235II, Fax 8.62136E+II, Email zhaoyd@aliyun.com

Received: June 20,2017 | Published: July 19, 2017
Abbreviations: LFS, li-fraumeni syndrome; VHL, von hippellindau

\section{Introduction}

The appearance of a new malignancy lesion after treatment for a previous cancer is generally diagnosed as a metastatic carcinoma; however, it is possible that the new malignancy may be a secondary primary malignancy, a phenomenon that has become increasingly more frequent, especially when the first primary malignancy was leukemia or esophageal carcinoma. ${ }^{1,2}$ The main reasons for the appearance of a secondary primary malignancy are presumed to be the following: 1) an increasing number of cancer patients exhibit longterm survival after proper treatments, ${ }^{3}$ and 2 ) the treatments (e.g., chemotherapy or radiotherapy) used to treat the first malignancy may result in a secondary primary malignancy. ${ }^{4,5}$ Therefore, the secondary primary malignancy generally occurs approximately 5-10 years (or even longer) after the first malignancy is treated. ${ }^{1}$ There are also some rare hereditary or familial syndromes (e.g., Li Fraumeni, Lynch, and Turcot syndrome) in which the concurrence of multiple cancers are much more common.

We recently treated one patient who was first diagnosed with carcinoma of the bladder, and a new tumor mass was found in her brain two months after the bladder cancer carcinectomy was performed. Interestingly, the post-operative pathological diagnosis was anaplastic astrocytoma, which excluded the possibility that the tumor resulted from brain metastasis of the primary bladder cancer. Moreover, because the interval between the occurrences of the two types of cancers was only two months, the glioma cannot be regarded as a common secondary primary malignancy. In addition, almost all of the patient's relatives had no similar medical histories, thus ruling out the possibility that the patient had one of the aforementioned familial syndromes. Here, we have chosen to describe our clinical experience with this patient for our peers because previous reports of similar cases are scarce and this type of case may easily be misdiagnosed and/or mistreated.

\section{Case presentation}

A 59-year-old female presented with a constant headache lasting for one week and was subsequently admitted. Two months before admission, she had suffered from invasive urothelial carcinoma, and a carcinectomy was performed to remove the bladder carcinoma (Figure 1A \& 1B). In addition, no relatives of the patient had previously exhibited a similar disease. The physical examination of the patient revealed only slight disorientation, and no other obviously positive nervous system signs were observed at admission. A cranial MRI scans revealed right temporal lobe lesions suggesting the presence of a glioma or metastatic tumor (Figure 1C). We resected the tumor mass via surgery, and the post-operative pathological diagnosis was anaplastic astrocytoma (WHO grade III, Figure 1D). The patient was then treated with combination chemotherapy (temozolomide and Nimustine) 6 times. An MRI rescan showed no obvious recurrence within 5 months of the operation, but a recurrence at the homolateral temporal horn appeared 7 months post-operation (Figure 1E). However, the patient refused further treatment and died 12 months after the glioma resection.

\section{Discussion}

According to monism, an intracranial lesion following a malignant tumor is generally initially suspected to be the result of brain metastases. However, brain metastases from lung cancer or breast cancer are much more common than those occurring from bladder cancer, which is very rare ${ }^{6}$ Moreover, the MRI scan did not reveal 
typical signs of intracranial metastases; therefore, we treated this patient as if the intracranial tumor was a primary glioma, a diagnosis that was confirmed by post-operative pathological results. Although, there are some reports suggesting that intracranial tumors can occur as secondary primary malignancies, ${ }^{4,7,8}$ the shortest interval between the two malignancies was greater than 12 months. ${ }^{8}$ However, the interval in our case was only two months, and the patient did not undergo any radiotherapy or chemotherapy during this period. Accordingly, our case was not that of a typical secondary primary malignancy. Rather, the two malignancies should be considered as two simultaneous primary malignancies because the symptoms of bladder carcinoma were generally more apparent and, thus, appeared earlier. In contrast, a glioma generally exhibits no typical symptoms and, thus, the malignancy was found later.

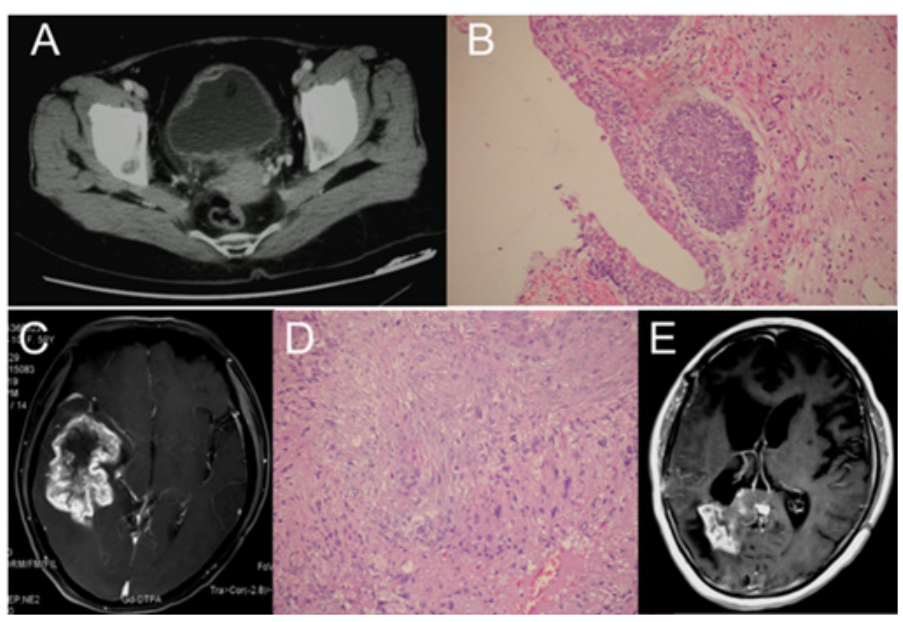

Figure I Radiological and pathological examinations.

A) A pelvic cavity $C T$ scan revealed an invasive lesion in the right anterior wall of the bladder (arrow); B) Post-operative pathological examination confirmed the diagnosis of high grade bladder cancer (200x); C) A cranial MRI scan showed a right temporal lobe lesion with uneven enhancement after injection of Gd-DTPA; D) The pathological diagnosis for the intracranial mass was anaplastic astrocytoma $(200 \times)$; E, the MRI scan 7 months after glioma resection showed a recurrence in the right temporal horn.

There are some rare cancer syndromes (e.g., Li Fraumeni, Lynch, and Turcot syndrome) in which the concurrence of multiple cancers are much more common. However, Li-Fraumeni syndrome (LFS), which is an autosomal dominant disorder, features the occurrence of breast cancer in young women and the occurrence of soft tissue sarcomas, osteosarcomas, brain tumors, acute leukemia, and/or adrenocortical tumors in children and young adults. ${ }^{9}$ There are significant differences in the age of onset between LFS and our case, as well as differences in the proband tumor types and degrees. Turcot's syndrome is another genetic disease characterized by the concurrence of primary brain tumors, colon cancers, and /or multiple colorectal adenomas at an early age. ${ }^{10}$ The age of onset in Turcot's syndrome is also quite different from the age of onset in our case. Furthermore, our patient in this case had no history of colon cancer or multiple colorectal adenomas and no similar familial history. Lynch syndrome, which is also called hereditary nonpolyposis colorectal cancer, is an autosomal dominant cancer-susceptibility syndrome caused by a germ-line mutation in one of the DNA-mismatch repair genes. ${ }^{11}$ Lynch syndrome exhibits an early age of onset and is associated with multiple types of cancer, including cancer of the colon and rectum, endometrium, ovary, small bowel, ureter, and renal pelvis. The symptoms of other cancer syndromes, such as Cowden syndrome, Werner syndrome, BeckwithWiedemann syndrome, and von Hippel-Lindau (VHL) hereditary cancer syndrome, were also inconsistent with the symptoms of our patient. Therefore, our case does not belong to any of these familial syndrome groups and is therefore likely to be a rare stand-alone sporadic case.

Are there correlations, causation or confounding factors involved in this case? This is an interesting problem. Based on the previous analysis, the patient seemed not to surfer from any of the cancer syndromes mentioned above. Perhaps the patient has some special genetic characteristics, making it easy to suffer from a variety of cancers, which needs to explore more deeply the pathological and clinical features.

As a clinical case, for primary gliomas, surgery is the preferred method of treatment; however, if the malignancy is the result of brain metastases, the value of surgery is sometimes controversial. In our case, the patient's diagnosis was primary glioma, and she underwent surgery and chemotherapy; however, her post-operative survival time was only 12 months. This result was worse than the results observed for common anaplastic astrocytoma, which may be related to the genetic features of the patient or a special biological characteristic of the cancer.

Previously, a bladder cancer patient with concurrent glioma has been rarely reported in the English literature. Our case reminds neurosurgeons that even when an intracranial lesion is found early after cancer occurs elsewhere in the body, the possibility of a primary glioma cannot be ruled out.

\section{Conflicts of interest}

Authors report no conflict of interest.

\section{Acknowledgments}

None.

\section{Funding}

None.

\section{References}

1. Neglia JP, Friedman DL, Yasui Y, et al. Second malignant neoplasms in five-year survivors of childhood cancer: childhood cancer survivor study. J Natl Cancer Inst. 2001;93(8):618-629.

2. R Poon RT, Law SY, Chu KM, et al. Multiple primary cancers in esophageal squamous cell carcinoma: incidence and implications. Ann Thorac Surg. 1998;65(6):1529-1534.

3. Hijiya N, Hudson MM, Lensing S, et al. Cumulative incidence of secondary neoplasms as a first event after childhood acute lymphoblastic leukemia. Jama. 2007;297(11):1207-1215.

4. Flickinger JC, Nelson PB, Martinez AJ, et al. Radiotherapy of nonfunctional adenomas of the pituitary gland. Results with long-term follow-up. Cancer. 1989;63(12):2409-2414.

5. Rimm IJ, Li FC, Tarbell NJ, et al. Brain tumors after cranial irradiation for childhood acute lymphoblastic leukemia. A 13-year experience from the Dana-Farber Cancer Institute and the Children's Hospital. Cancer 1987;59(8):1506-1508.

6. Steinfeld AD, Zelefsky M. Brain metastases from carcinoma of bladder. Urology. 1987;29(4):375-376.

7. Hawkins MM, Draper GJ, Kingston JE. Kingston, Incidence of second primary tumours among childhood cancer survivors. British journal of cancer. 1987;56(3):339-347. 
8. Kingston JE, Hawkins MM, Draper GJ, et al. Patterns of multiple primary tumours in patients treated for cancer during childhood. British journal of cancer. 1987;56(3):331-338.

9. Hisada M, Garber JE, Fung CY, et al. Multiple primary cancers in families with Li-Fraumeni syndrome. J Natl Cancer Inst. 1998;90(8):606-611.
10. Miyaki M, Nishio J, Konishi M, et al. Utsunomiya, Drastic genetic instability of tumors and normal tissues in Turcot syndrome. Oncogene. 1997;15(23):2877-2881.

11. Schmeler KM, Lynch HT, Chen LM, et al. Prophylactic surgery to reduce the risk of gynecologic cancers in the Lynch syndrome. $N$ Engl J Med. 2006;354(3):261-269. 